\title{
Editorial: Plant Phytochromes: From Structure to Signaling and Beyond
}

\author{
András Viczián ${ }^{1 *}$, Cornelia Klose ${ }^{2}$, Andreas Hiltbrunner ${ }^{2,3}$ and Ferenc Nagy ${ }^{1}$ \\ ${ }^{1}$ Biological Research Centre, Institute of Plant Biology, Laboratory of Photo- and Chronobiology, Eötvös Loránd Research \\ Network (ELKH), Szeged, Hungary, ${ }^{2}$ Institute of Biology II, University of Freiburg, Freiburg im Breisgau, Germany, ${ }^{3}$ Centre for \\ Biological Signalling Studies (BIOSS) and Centre for Integrative Biological Signalling Studies (CIBSS), University of Freiburg, \\ Freiburg im Breisgau, Germany
}

Keywords: photoreceptor, phytochrome, photomorphogenesis, deetiolation, light signaling

\section{Editorial on the Research Topic}

\section{Plant Phytochromes: From Structure to Signaling and Beyond}

\section{OPEN ACCESS}

Edited and reviewed by: Kris Vissenberg,

University of Antwerp, Belgium

${ }^{*}$ Correspondence: András Viczián viczian.andras@brc.hu

Specialty section: This article was submitted to Plant Cell Biology, a section of the journal

Frontiers in Plant Science

Received: 08 November 2021 Accepted: 23 November 2021 Published: 09 December 2021

Citation:

Viczián A, Klose C, Hiltbrunner A and Nagy F (2021) Editorial: Plant Phytochromes: From Structure to Signaling and Beyond.

Front. Plant Sci. 12:811379. doi: 10.3389/fpls.2021.811379
Light is the major source of energy for plants and thus light sensing is vital for their survival. Specialized photoreceptor molecules transform the energy of the photons to chemical and biological signals that control growth and development and the adaptation to changes in the environment. Phytochromes are synthesized in their inactive (Pr) conformer and red $\left(\lambda_{\max }=\right.$ $660 \mathrm{~nm}$ ) light illumination can convert them to the Pfr form (Rockwell et al., 2006). Pfr is the biologically active form of phytochromes, it initiates various signaling pathways that lead to a suite of responses known as photomorphogenic development. Pfr conversion back to Pr can be triggered by far-red $\left(\lambda_{\max }=730 \mathrm{~nm}\right)$ photons or by a spontaneous reversion to the lower-energy Pr state by the so-called thermal relaxation (Klose et al., 2020). There are five phytochromes (phyA-phyE) expressed in the widely used model plant Arabidopsis thaliana (Mathews, 2010). Since the discovery of phytochrome-mediated responses in plants (Flint, 1936; Borthwick et al., 1952) our knowledge has expanded toward multiple directions. This Frontiers Research Topic gives insights into the current state of some of these aspects of phytochrome research.

Phytochrome signaling pathways control about $10 \%$ of the Arabidopsis transcriptome (Ma et al., 2001) and these transcriptional responses are essential for environmental adaptation. Whereas, initial research efforts focused purely on examining phytochrome-dependent signaling in the context of light responses, it later became clear that phytochromes integrate light with other environmental signals such as temperature (Jung et al., 2016; Legris et al., 2019), cold stress (Franklin and Whitelam, 2007), drought stress (González et al., 2012), and pathogen attack (de Wit et al., 2013). In a review paper, Kim et al. point out that phyB, the most abundant phytochrome in light-grown plants, plays the major role in these responses. The authors summarize how phyB takes part in plant responses to diverse environmental signals and give an insight into the complexity of these signaling pathways.

Pardi and Nusinow focused on a special aspect of phytochrome signaling. It is well-established that active phytochromes translocate from the cytoplasm into the nucleus and compartmentalize into distinct molecular complexes, so-called photobodies (Yamaguchi et al., 1999; Kircher et al., 2002; Van Buskirk et al., 2012). Photobodies are essential for proper signaling but their function, composition, and biogenesis have remained unclear. Pardi and Nusinow summarized our recent knowledge about (i) possible biological roles of photobodies, (ii) protein components regulating their formation and (iii) mechanisms of photobody biogenesis including a discussion about the idea that phyB containing photobodies may be the result of liquid-liquid phase separation, similar to photobodies formed by cryptochrome 2 (Wang et al., 2021). 
Although phytochromes were originally identified in seed plants (Butler et al., 1959) we now know that not only plants but also different bacteria and fungi possess phytochromes (Karniol and Vierstra, 2003). Lamparter et al. focused in their review article on Agrobacterium phytochromes. Agrobacterium fabrum contains two phytochromes called Agp1 and Agp2. The authors list the original publications leading to the discovery of Agps and summarize our recent knowledge about Agp protein structure and functions. These studies are a valuable addition to plant phytochrome research, not only from an evolutionary perspective. The advances made on Agrobacterium phytochrome structure determination support our efforts made on plant phytochromes, because crystallization of Agps is technically less challenging than that of plant PHYs.

Wahlgren et al. developed a methodological approach focusing on this problem. They examined the structure of recombinant Arabidopsis phyA using cryo-electron microscopy. The $17 \AA$ resolution achieved is not exceptionally high, showing clear limitations of this approach, but the data were obtained using homodimers from near-homogenous and photochemically active protein preparations. The authors parallel their results with earlier studies performed on bacterial phytochromes and discuss further possible applications of the method.

Posttranslational modifications (PTM) occur during or after translation resulting in a covalent attachment of a moiety modifying the activity of the target protein. Phytochromes are targets of diverse PTMs; among them, phosphorylation was identified decades ago (Quail et al., 1978; Hunt and Pratt, 1980). This PTM is reversible and fine-tunes light signaling by changing the amount of available active phytochrome molecules (Stockhaus et al., 1992; Medzihradszky et al., 2013; Nito et al., 2013; Viczián et al., 2020). The two obvious regulatory steps of the phosphorylation state of the available phytochrome pools are phosphorylation and dephosphorylation. It was demonstrated that phytochromes act as serine/threonine kinases autophosphorylating themselves (Shin et al., 2016). The work of Hoang et al. added further details to the picture. The authors showed that specific missense mutations in the Avena sativa phyA result in increased phytochrome kinase activity. These mutant PHYAs trigger hypersensitive photoresponses when expressed in transgenic Arabidopsis plants, indicating that there is a direct positive correlation between the kinase activity of the phytochromes and the observable light responses in planta.

Whereas, external kinases that phosphorylate phytochromes have not been identified so far, phosphatases that dephosphorylate PHYs have already been described (Kim et al., 2002; Ryu et al., 2005; Phee et al., 2008). One of them, Phytochrome-Associated Protein Phosphatase 5 (PAPP5) dephosphorylates active $\mathrm{Pfr}$ phytochromes and enhances phytochrome-mediated responses (Ryu et al., 2005). von Horsten and Essen were able to crystallize recombinant PAPP5 and determine its structure at $3 \AA$ resolution. The authors could examine the effect of different compounds on PAPP5 activity, analyze the interaction details and dynamics of PAPP5 with phospho-site mimicking mutant PHYB molecules focusing on the positional arrangement of PAPP5's known domains.
Furthermore, this study indicates that the activation mode of PAPP5 is similar to that of its mammalian counterparts. The sterical consequences of PAPP5 activation by arachidonic acid suggest an exciting novel regulatory pathway linking plant defense mechanisms and phytochrome regulation by dephosphorylation, but this finding needs to be further validated in the future.

This Research Topic collected studies tackling interesting issues of the phytochrome field and rising fundamental questions that need to be addressed in the future. (i) Although the consequences of phosphorylation at certain amino acids on the function of phytochrome proteins were examined, the detailed phytochrome "phospho-map" is still missing. Furthermore, at the moment we do not know how the interplay of different PTMs fine-tune phytochrome signaling. (ii) We also do not know to what extent PHY autophosphorylation, and the activity of different kinases and phosphatases are responsible for the actual phospho-state of the available phytochrome pool and the performance of phytochrome signaling. Further targets of phytochrome kinase activity and its significance on the signaling process also need to be identified in the future. (iii) Two studies in this Research Topic focused on the molecular structure of phytochromes and their partners indicating the continued interest in the subject. Despite recent advances, we still need to achieve high resolution structural models of full length plant phytochromes in $\mathrm{Pr}$ and $\mathrm{Pfr}$ to understand the overall structural rearrangements during photoconversion. (iv) Hopefully, this structural information will allow designing structure-function models for phytochrome signaling that could be tested also experimentally. (v) Longlasting efforts from the scientific community resulted in the identification of phytochrome interacting partners. We expect that examining the phytochrome containing photobodies will help us to understand the interaction dynamics of phytochromes with their signaling partners and to map these signaling networks in details. (vi) Furthermore, it is crucial to examine the role of phytochromes not only in light signaling but also in signal interactions of interconnecting pathways in order to understand how plants respond to complex environmental stimuli.

We, the Guest Editors of this article collection, believe that the set of papers published here stimulates further discussions and initiates studies answering the pending questions.

\section{AUTHOR CONTRIBUTIONS}

$\mathrm{AV}$ wrote the manuscript. $\mathrm{CK}, \mathrm{AH}$, and $\mathrm{FN}$ corrected and contributed to the text. All authors agreed in the final version.

\section{FUNDING}

This work was supported by grants from the Hungarian Scientific Research Fund (OTKA, K-132633 for AV and FN and K138022 for AV) and the German Research Foundation (DFG) under Germany's Excellence Strategy (EXC-2189-Project ID 390939984, project $\mathrm{C} 1$ to $\mathrm{AH})$. 


\section{REFERENCES}

Borthwick, H. A., Hendricks, S. B., Parker, M. W., Toole, E. H., and Toole, V. K. (1952). A reversible photoreaction controlling seed germination. Proc. Natl. Acad. Sci. U.S.A. 38, 662-666. doi: 10.1073/pnas.38.8.662

Butler, W. L., Norris, K. H., Siegelman, H. W., and Hendricks, S. B. (1959). Detection, assay, and preliminary purification of the pigment controlling photoresponsive development of plants. Proc. Natl. Acad. Sci. U.S.A. 45, 1703-1708. doi: 10.1073/pnas.45.12.1703

de Wit, M., Spoel, S. H., Sanchez-Perez, G. F., Gommers, C. M. M., Pieterse, C. M. J., Voesenek, L. A. C. J., et al. (2013). Perception of low red:far-red ratio compromises both salicylic acid- and jasmonic acid-dependent pathogen defences in Arabidopsis. Plant J. 75, 90-103. doi: 10.1111/tpj.12203

Flint, L. H. (1936). The action of radiation of specific wave-lengths in relation to the germination of light-sensitive lettuce seed. Proc. Int. Seed Test. Assoc. 8, 1-4.

Franklin, K. A., and Whitelam, G. C. (2007). Light-quality regulation of freezing tolerance in Arabidopsis thaliana. Nat. Genet. 39, 1410-1413. doi: $10.1038 / \mathrm{ng} .2007 .3$

González, C. V., Ibarra, S. E., Piccoli, P. N., Botto, J. F., and Boccalandro, H. E. (2012). Phytochrome B increases drought tolerance by enhancing ABA sensitivity in Arabidopsis thaliana. Plant Cell Environ. 35, 1958-1968. doi: 10.1111/j.1365-3040.2012.02529.x

Hunt, R. E., and Pratt, L. H. (1980). Partial characterization of undegraded oat phytochrome. Biochemistry 19, 390-394. doi: 10.1021/bi00543a022

Jung, J.-H., Domijan, M., Klose, C., Biswas, S., Ezer, D., Gao, M., et al. (2016). Phytochromes function as thermosensors in Arabidopsis. Science 354, 886-889. doi: $10.1126 /$ science.aaf600

Karniol, B., and Vierstra, R. D. (2003). The pair of bacteriophytochromes from Agrobacterium tumefaciens are histidine kinases with opposing photobiological properties. Proc. Natl. Acad. Sci. U.S.A. 100, 2807-2812. doi: 10.1073/pnas.0437914100

Kim, D.-H., Kang, J.-G., Yang, S.-S., Chung, K.-S., Song, P.-S., and Park, C.-M. (2002). A phytochrome-associated protein phosphatase 2A modulates light signals in flowering time control in Arabidopsis. Plant Cell 14, 3043-3056. doi: $10.1105 /$ tpc.005306

Kircher, S., Gil, P., Kozma-Bognar, L., Fejes, E., Speth, V., Husselstein-Muller, T., et al. (2002). Nucleocytoplasmic partitioning of the plant photoreceptors phytochrome A, B, C, D, and E is regulated differentially by light and exhibits a diurnal rhythm. Plant Cell 14, 1541-1555. doi: 10.1105/tpc.001156

Klose, C., Nagy, F., and Schäfer, E. (2020). Thermal reversion of plant phytochromes. Mol. Plant 13, 386-397. doi: 10.1016/j.molp.2019.12.004

Legris, M., Ince, Y. Ç., and Fankhauser, C. (2019). Molecular mechanisms underlying phytochrome-controlled morphogenesis in plants. Nat. Commun. 10:5219. doi: 10.1038/s41467-019-13045-0

Ma, L., Li, J., Qu, L., Hager, J., Chen, Z., Zhao, H., et al. (2001). Light control of Arabidopsis development entails coordinated regulation of genome expression and cellular pathways. Plant Cell 13, 2589-2607. doi: 10.1105/tpc.010229

Mathews, S. (2010). Evolutionary studies illuminate the structural-functional model of plant phytochromes. Plant Cell 22, 4-16. doi: 10.1105/tpc.109.072280

Medzihradszky, M., Bindics, J., Adam, E., Viczian, A., Klement, E., Lorrain, S., et al. (2013). Phosphorylation of phytochrome B inhibits light-induced signaling via accelerated dark reversion in Arabidopsis. Plant Cell 25, 535-544. doi: $10.1105 /$ tpc. 112.106898
Nito, K., Wong, C. C. L., Yates, J. R., and Chory, J. (2013). Tyrosine phosphorylation regulates the activity of phytochrome photoreceptors. Cell Rep. 3, 1970-1979. doi: 10.1016/j.celrep.2013.05.006

Phee, B.-K., Kim, J.-I., Shin, D. H., Yoo, J., Park, K.-J., Han, Y.-J., et al. (2008). A novel protein phosphatase indirectly regulates phytochrome-interacting factor 3 via phytochrome. Biochem. J. 415, 247-255. doi: 10.1042/BJ20071555

Quail, P. H., Briggs, W. R., and Pratt, L. H. (1978). In vivo phosphorylation of phytochrome. Carnegie Institution Yearbook 1978, 342-344.

Rockwell, N. C., Su, Y.-S., and Lagarias, J. C. (2006). Phytochrome structure and signaling mechanisms. Annu. Rev. Plant Biol. 57, 837-858. doi: 10.1146/annurev.arplant.56.032604.144208

Ryu, J. S., Kim, J.-I., Kunkel, T., Kim, B. C., Cho, D. S., Hong, S. H., et al. (2005). Phytochrome-specific type 5 phosphatase controls light signal flux by enhancing phytochrome stability and affinity for a signal transducer. Cell 120, 395-406. doi: 10.1016/j.cell.2004.12.019

Shin, A.-Y., Han, Y.-J., Baek, A., Ahn, T., Kim, S. Y., Nguyen, T. S., et al. (2016). Evidence that phytochrome functions as a protein kinase in plant light signalling. Nat. Commun. 7:11545. doi: 10.1038/ncomms11545

Stockhaus, J., Nagatani, A., Halfter, U., Kay, S., Furuya, M., and Chua, N. H. (1992). Serine-to-alanine substitutions at the amino-terminal region of phytochrome A result in an increase in biological activity. Genes Dev. 6, 2364-2372. doi: 10.1101/gad.6.12a.2364

Van Buskirk, E. K., Decker, P. V., and Chen, M. (2012). Photobodies in light signaling. Plant Physiol. 158, 52-60. doi: 10.1104/pp.111.186411

Viczián, A., Ádám, É., Staudt, A.-M., Lambert, D., Klement, E., Romero Montepaone, S., et al. (2020). Differential phosphorylation of the N-terminal extension regulates phytochrome B signaling. New Phytol. 225, 1635-1650. doi: $10.1111 /$ nph.16243

Wang, X., Jiang, B., Gu, L., Chen, Y., Mora, M., Zhu, M., et al. (2021). A photoregulatory mechanism of the circadian clock in Arabidopsis. Nat Plants 7, 1397-1408. doi: 10.1038/s41477-021-01002-Z

Yamaguchi, R., Nakamura, M., Mochizuki, N., Kay, S. A., and Nagatani, A. (1999). Light-dependent translocation of a phytochrome B-GFP fusion protein to the nucleus in transgenic Arabidopsis. J. Cell Biol. 145, 437-445. doi: $10.1083 /$ jcb.145.3.437

Conflict of Interest: The authors declare that the research was conducted in the absence of any commercial or financial relationships that could be construed as a potential conflict of interest.

Publisher's Note: All claims expressed in this article are solely those of the authors and do not necessarily represent those of their affiliated organizations, or those of the publisher, the editors and the reviewers. Any product that may be evaluated in this article, or claim that may be made by its manufacturer, is not guaranteed or endorsed by the publisher.

Copyright (c) 2021 Viczián, Klose, Hiltbrunner and Nagy. This is an open-access article distributed under the terms of the Creative Commons Attribution License (CC $B Y)$. The use, distribution or reproduction in other forums is permitted, provided the original author(s) and the copyright owner(s) are credited and that the original publication in this journal is cited, in accordance with accepted academic practice. No use, distribution or reproduction is permitted which does not comply with these terms. 